October 13, 2018

\title{
Spin-electric Berry phase shift in triangular molecular magnets
}

\author{
Vahid Azimi Mousolou, ${ }^{1}$ C. M. Canali, ${ }^{2}$ and Erik Sjöqvist ${ }^{3}$ \\ ${ }^{1}$ Department of Mathematics, Faculty of Science, \\ University of Isfahan, Box 81745-163 Isfahan, Iran \\ ${ }^{2}$ Department of Physics and Electrical Engineering, \\ Linnaeus University, SE-391 82 Kalmar, Sweden \\ ${ }^{3}$ Department of Physics and Astronomy, Uppsala University, Box 516, SE-751 20 Uppsala, Sweden
}

\begin{abstract}
We propose a Berry phase effect on the chiral degrees of freedom of a triangular magnetic molecule. The phase is induced by adiabatically varying an external electric field in the plane of the molecule via a spin-electric coupling mechanism present in these frustrated magnetic molecules. The Berry phase effect depends on spin-orbit interaction splitting and on the electric dipole moment. By varying the amplitude of the applied electric field, the Berry phase difference between the two spin states can take any arbitrary value between zero and $\pi$, which can be measured as a phase shift between the two chiral states by using spin-echo techniques. Our result can be used to realize an electric field induced geometric phase-shift gate acting on a chiral qubit encoded in the ground state manifold of the triangular magnetic molecule.
\end{abstract}

PACS numbers: 03.65.Vf, 75.50.Xx, 75.75-c, 03.67.Lx

\section{INTRODUCTION}

Berry's phase, originally discovered $\underline{\underline{1}}$ for a nondegenerate pure quantum state evolving adiabatically in a cyclic fashion, has been subsequently extended to nonadiabatic evolution ${ }^{2.3}$, the evolution of degenerate quantum states $^{4}$, and mixed states $5, \underline{6}$. In parallel to the theoretical development, these phases have been demonstrated experimentally in a wide variety of contexts, including optical ${ }^{\underline{7}}$, molecular ${ }^{\frac{8}{}}$, and solid-state ${ }^{\frac{9}{}}$ systems. The properties of the Berry phase make it an essential unifying concept in the physical sciences $\underline{10}$.

The emerging and partly overlapping fields of molecular quantum spintronics $\frac{11}{1}$ and magnon spintronics ${ }^{12}$ offer a promising approach to design devices for information storage, transport and processing. In this development, magnetic molecules $(\mathrm{MMs})^{13}$ play a central role. MMs possess rich quantum properties, which can be chemically engineered. There has been considerable recent interest in MMs since they all have, in contrast to, e.g., nanoparticles, identical properties, which is an important advantage for the realization of scalable ensembles of quantum computation entities. Antiferromagnetic triangular molecules such as $\left\{\mathrm{Cu}_{3}\right\}$ complexes (e.g., $\left.\mathrm{Na}_{12}\left[\mathrm{Cu}_{3}\left(\mathrm{AsW}_{9} \mathrm{O}_{33}\right)_{2} \cdot 3 \mathrm{H}_{2} \mathrm{O}\right] \cdot 32 \mathrm{H}_{2} \mathrm{O}\right) \stackrel{14}{\underline{4}}$ are a special class of MMs particularly suitable for quantum control and manipulation. Due to the lack of inversion symmetry, these triangular MMs display an effective spin-electric coupling mechanism $15-17$ acting within their quasi-degenerate chiral ground state. This provides a proper and applicable way to do quantum information processing with spin systems, since electric fields are simpler to apply and control at small spatial scales and short time scales, compared to magnetic fields $\underline{16}, 17$. For these reasons, electrical control of electron and nuclear spin qubits is a subject intensively investigated, not only in
$\mathrm{MMs}^{18}$, but also in semiconductor quantum dots containing single electrons $\stackrel{19}{ }$, and in devices consisting of single impurities in a semiconductor host, e.g., $\mathrm{P}$-donors in $\mathrm{Si}^{20}$, as originally proposed by $\mathrm{Kane}^{21}$.

Laser-induced nonadiabatic (femtosecond) spin dynamics in isolated triangular clusters, such as $\mathrm{Co}_{3}^{+}(\mathrm{EtOh}), \quad \mathrm{Co}_{3}^{+}(\mathrm{EtOh}), \quad \mathrm{Ni}_{3}\left(\mathrm{CH}_{3} \mathrm{OH}\right), \quad$ and $\mathrm{Co}^{+}\left(\mathrm{CH}_{3} \mathrm{OH}\right)$ have been studied recently both theoretically (using advanced ab-initio methods) and experimentally in molecular beam experiments 22,23 . These tri-nuclear transition-metal clusters are different from the antiferromagnetic triangular molecules considered in the present paper. Their spin properties make them closely resemble single-molecule magnets $(\mathrm{SMM}) \underline{24}$. Nevertheless these studies show that efficient electric manipulations of the molecular spin density, in this case mediated by spin-orbit interaction, is feasible. In principle, similar molecular beam experiments could be extended also to triangular antiferromagnetic molecules such as $\mathrm{Cu}_{3}$, where a coupling between spin chiral states and the electric field is present even in the absence of spin-orbit coupling.

In this paper we show that in a triangular antiferromagnetic MM subject to a time-dependent external electric field, the spin-electric coupling induces a Berry phase in the spin-chiral ground state manifold. We use applied electric field pulses in the presence of a static magnetic field to realize a conditional dynamics of the system. We show that Berry phases with arbitrary values between zero to $\pi$ on the chiral degree of freedom (chiral qubit) can be achieved by adiabatically varying the electric field in the plane of the molecule, and can be measured by using the spin-echo technique. The Berry phase shift depends on the effective spin-orbit interaction and electric dipole moment of the MM, which are the two most important quantities that control the spin-electric coupling 
mechanism. In the adiabatic limit, the ratio of these two quantities can be determined by measuring the Berry phase shift as a function of the electric field amplitude. The Berry phase can be used to implement a phase-shift gate, with an arbitrary phase, acting on the chiral part of the ground state manifold of the triangular MM, which encodes a single chiral qubit. These gates are electric field generated and geometric, two key ingredients to realize flexible and coherent switching, which is needed for the implementation of efficient quantum processors.

The outline of the paper is as follows. In the next section, we provide a brief introduction to spin-electric coupling in triangular MMs and their ground state manifold. In Sec. III we describe the conditional dynamics with respect to the spin-chirality decomposition of the system. We demonstrate how it can be used to test the geometric Berry phase effect on the chirality in Sec. IV] The paper is summarized in Sec. $\mathrm{V}$

\section{EFFECTIVE QUBIT SYSTEM}

The degeneracy of the ground state (GS) of spin rings containing an odd number of antiferromagnetically coupled half-integer spins make them a suitable candidate for quantum information processing. In particular, oddnumber rings of half-odd integer spins satisfy the conditions, which allow for spin state manipulation via pulsed electric fields $\frac{17}{}$. The simplest nontrivial class of such a spin system is a triangular ring of $s=\frac{1}{2}$ spins, e.g., $\mathrm{Cu}_{3}, \mathrm{~V}_{15}, \mathrm{Co}_{3}$. As depicted in Fig. [1 the magnetic core of such MMs consists of three $s=\frac{1}{2}$ spins positioned at the vertices of an equilateral triangle and coupled by an antiferromagnetic Heisenberg exchange interaction. In the absence of external fields, the system can be described by the spin Hamiltonian $\frac{17}{17}$

$$
\begin{aligned}
H= & \sum_{k=1}^{3} J_{k, k+1} \mathbf{s}_{k} \cdot \mathbf{s}_{k+1} \\
& +\sum_{k=1}^{3} \mathbf{D}_{k, k+1} \cdot\left(\mathbf{s}_{k} \times \mathbf{s}_{k+1}\right),
\end{aligned}
$$

with a periodic boundary condition, where the first and fourth sites are identified. Here, $\mathbf{s}_{k}$ is a spin- $\frac{1}{2}$ vector operator localized at site $k$. In Eq. (11), the first term is an isotropic Heisenberg interaction with antiferromagnetic exchange couplings $J_{k, k+1}$, and the second term is an antisymmetric Dzyaloshinsky-Moriya (DM) interaction 26,27 . Under the assumption, that the magnetic Hamiltonian of the molecule is invariant under point group $D_{3 h}$, its parameters satisfy the constraints

$$
J_{k, k+1}=J, \quad \mathbf{D}_{k, k+1}=\left(0,0, D^{z}\right), \quad k=1,2,3 .
$$

The GS manifold of these frustrated MMs is given by two degenerate total spin $S=\frac{1}{2}$ doublets $\left(S_{z}= \pm \frac{1}{2}\right)$ of opposite spin chirality $\chi= \pm 1$. Explicitly, the GSspace is spanned by the following linearly independent

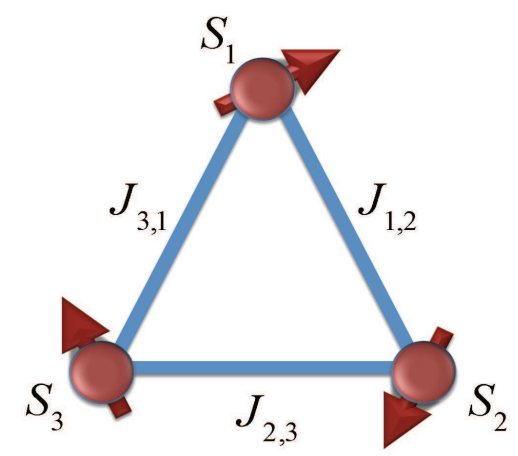

FIG. 1. Schematic geometry of triangular molecular magnets.

quadruplet,

$$
\begin{aligned}
\left| \pm 1,+\frac{1}{2}\right\rangle & =\frac{1}{\sqrt{3}}\left[|\downarrow \uparrow \uparrow\rangle+\eta_{ \pm}|\uparrow \downarrow \uparrow\rangle+\eta_{\mp}|\uparrow \uparrow \downarrow\rangle\right], \\
\left| \pm 1,-\frac{1}{2}\right\rangle & =\frac{1}{\sqrt{3}}\left[|\uparrow \downarrow \downarrow\rangle+\eta_{ \pm}|\downarrow \uparrow \downarrow\rangle+\eta_{\mp}|\downarrow \downarrow \uparrow\rangle\right], \\
\eta_{ \pm} & =e^{ \pm i 2 \pi / 3}
\end{aligned}
$$

constructed by symmetry adapted linear combination of various possible spin configurations. The quantum basis states given in Eq. (3) are simultaneous eigenvectors of the $z$-component of chirality and total spin operators, i.e., $C_{z}$ and $S_{z}$, respectively, where the components of the chirality vector operator read

$$
\begin{aligned}
C_{x} & =-\frac{2}{3}\left(\mathbf{s}_{1} \cdot \mathbf{s}_{2}-2 \mathbf{s}_{2} \cdot \mathbf{s}_{3}+\mathbf{s}_{3} \cdot \mathbf{s}_{1}\right), \\
C_{y} & =\frac{2}{\sqrt{3}}\left(\mathbf{s}_{1} \cdot \mathbf{s}_{2}-\mathbf{s}_{3} \cdot \mathbf{s}_{1}\right), \\
C_{z} & =\frac{4}{\sqrt{3}} \mathbf{s}_{1} \cdot\left(\mathbf{s}_{2} \times \mathbf{s}_{3}\right) .
\end{aligned}
$$

One can verify that the chiral operators define the same algebra as the spin-half operators. Namely, $C_{k}, k=$ $x, y, z$, in the chiral basis states $|\chi= \pm 1\rangle$ are the same as Pauli matrix components, and thus $\left[C_{k}, C_{l}\right]=$ $2 i \sum_{m} \epsilon_{k l m} C_{m}$, with $\epsilon_{k l m}$ being the Levi-Civita symbol.

The energy gap $\Delta_{J}$ between the GS manifold and the first excited state, the $S=\frac{3}{2}$ quadruplet, is typically of the order of $1 \mathrm{meV}: \underline{28}$ The spin-orbit induced DM interaction lifts the degeneracy between the two chiral doublets with a splitting $\Delta_{\mathrm{SO}} \leq 0.1 \Delta_{J} \underline{16,29}$. What makes these triangular MMs interesting for quantum manipulation is that an electric field in the $x y$-plane of the molecule couples the two GS doublets of opposite chirality, due to the lack of inversion symmetry $16,17,30$.

In the presence of external electric (E) and magnetic (B) fields, the dynamics of the GS-space spanned by the basis states given in Eq. (3) is described by the effective low-energy spin Hamiltonian 16

$$
H_{\mathrm{eff}}=\Delta_{\mathrm{SO}} C_{z} S_{z}+p \mathbf{E}^{\prime} \cdot \mathbf{C}_{\|}+\mathbf{B} \overline{\bar{g}} \cdot \mathbf{S}
$$

with $\mathbf{C}_{\|}=\left(C_{x}, C_{y}, 0\right)$ and $\mathbf{S}=\left(S_{x}, S_{y}, S_{z}\right)$ being the chirality and spin vector operators, respectively. $\mathbf{E}^{\prime}=$ 
$\mathcal{R}_{z}(\alpha) \mathbf{E}$ is the electric field $\mathbf{E}$ rotated about the $z$ axis by an angle $\alpha=7 \pi / 6-2 \beta$ with $\beta$ being the angle between the in-plane component of the electric field $\mathbf{E}$ and a vector pointing from site 1 to 2 . Due to the symmetry of the molecule $\overline{\bar{g}}=\operatorname{diag}\left\{g_{\|}, g_{\|}, g_{\perp}\right\}$. The parameter $p$ has the units of an electric dipole moment, and it gives the strength of the effective coupling between the two states with opposite chirality brought about by the electric field. In $\mathrm{Cu}_{3} \mathrm{MM} p$ is not small ${ }^{30}$, and for typical electric fields generated by a scanning tunneling microscope $(\mathrm{STM})\left(\approx 10^{2} \mathrm{kV} / \mathrm{cm}\right)$ the spin-chirality manipulation (Rabi) time is $10-10^{3} \mathrm{ps} \frac{16.30}{}$.

We conclude this section with a discussion on the validity of the effective spin Hamiltonian given in Eq. (1), satisfying the constraints of Eq. (2) imposed by the $D_{3 h}$ symmetry of the molecule. It is known ${ }^{31}$ that equilateral triangular molecules with an odd number of electrons undergo a Jahn-Teller (JT) distortion that reduces the $D_{3 h}$ symmetry. Typically the deformation makes one of the sides slightly shorter or longer, leading to an isosceles triangle with $D_{2 v}$ symmetry. For $\mathrm{Cu}_{3}$ complexes and other triangular molecules the deformation is found to be very tiny both experimentally $\underline{14}$ and theoretically $\underline{32}$ (the side change is of the order of $0.001 \AA$ for $\mathrm{Cu}_{3}$ complexes ${ }^{14}$ ), and it is usually neglected ${ }^{16}$. The JT distortion mechanism lifts the chiral degeneracy of the ground state, even in the absence of spin-orbit coupling. We can describe this effect within the spin Hamiltonian approach by adding to Eq. (1) the JT-induced correction

$$
\delta H_{\mathrm{JT}}=\sum_{k=1}^{3} \delta J_{k, k+1} \mathbf{s}_{k} \cdot \mathbf{s}_{k+1},
$$

where $\delta J_{k, k+1}$ are the modifications in the exchange constants caused the changes in the bond lengths. Since the deformation is tiny, we typically have $\delta J_{k, k+1} / J \ll 1$, where $J$ is the coupling constant for the equilateral nuclear configuration. For example, for the case of $\mathrm{Cu}_{3}$, where $J \approx 1 \mathrm{meV}, \delta J_{k, k+1}<0.1 \mathrm{meV}$. For a distortion down to an isosceles triangle, two of these $\delta J_{k, k+1}$ are equal. Since $\delta H_{\mathrm{JT}}$ is still rotationally invariant in spin space, the total spin of the total Hamiltonian remains a good quantum number. Therefore, $\delta H_{\mathrm{JT}}$ couples the two $S=\frac{1}{2}$ chiral GS states, lifting the degeneracy of the unperturbed Hamiltonian, but does not couple these to the $S=\frac{3}{2}$ excited state quadruplet. Using the definition of the chiral vector operator in Eq. (4), one can see that $\delta H_{\mathrm{JT}}$ can be formally rewritten as

$$
\delta H_{\mathrm{JT}}=p \mathbf{E}_{\mathrm{JT}} \cdot \mathbf{C}_{\|},
$$

where $\mathbf{E}_{\mathrm{JT}}$ is an internal electric field describing the JT deformation of the molecule ${ }^{16}$. Here the components of the vector $p \mathbf{E}_{\text {JT }}$ can be easily related to the parameters $\delta J_{k, k+1}$.

Since the JT reduces the symmetry of the molecule to $D_{2 v}$, the DM term is also modified by the JT distortion. This effect can also be studied by adding an appropriate spin Hamiltonian to Eq. (1). This perturbation in general breaks rotation symmetry in spin space, and besides coupling the two GS $S=\frac{1}{2}$ chiral states with each other, also couples these to the $S=\frac{3}{2}$ excited state. However, since the DM exchange constant for the unperturbed system is at least one-order of magnitude smaller than isotropic $J$, its change induced by the $J T$ distortion is typically small with respect to $\delta J_{k, k+1}$. Therefore, we expect the effect of this JT-induced perturbation on the low-energy levels of the system to be considerably smaller than the one caused by Eq. (7).

In conclusion, the main effect of the JT distortion can be described by the presence of a small intrinsic static in-plane electric field, which is combined to the applied external electric field $\mathbf{E}^{\prime}$ in Eq. (5).

Note finally that for an isolated triangular molecule in the gas phase, there are three equivalent JT distorted (isosceles) configurations, all with the same GS energy but separated by an energy barrier. It is then possible for the system to quantum tunnel from any one of these configurations to the other two. This phenomenon, known as the dynamical JT effect, can effectively restore the original $D_{3 h}$ symmetry of the molecule ${ }^{33}$, provided that the energy barrier separating the three JT distorted states is small compared to perturbations that couple them.

\section{CONDITIONAL DYNAMICS OF THE SYSTEM}

In the presence of a static magnetic field in the $z$ direction, an electric field $\mathbf{E}$ induces transitions only within each eigensubspace of $S_{z}$ of the chiral state manifold. This implies that the effective spin Hamiltonian in Eq. (5) can be decomposed into two parts corresponding to the eigenvalues $\pm \frac{1}{2}$ of $S_{z}$. Therefore, the system describes two independent chiral components, decoupled from each other and split by the external magnetic field (see Fig. 2). For a time-dependent oscillating electric field $\mathbf{E}(t)$, the corresponding Hamiltonian takes the form

$$
\begin{aligned}
H_{\mathrm{eff}}(t)= & \frac{\hbar}{2}\left[\Omega_{0} I+\boldsymbol{\Omega}_{+}(t) \cdot \boldsymbol{\sigma}\right] \otimes\left|+\frac{1}{2}\right\rangle\left\langle+\frac{1}{2}\right| \\
& +\frac{\hbar}{2}\left[-\Omega_{0} I+\boldsymbol{\Omega}_{-}(t) \cdot \boldsymbol{\sigma}\right] \otimes\left|-\frac{1}{2}\right\rangle\left\langle-\frac{1}{2}\right|,
\end{aligned}
$$

where $\hbar \Omega_{0}=g_{\perp} B_{z}$,

$$
\hbar \boldsymbol{\Omega}_{ \pm}(t)=\left(p \mathcal{E} \cos (\omega t+\phi), p \mathcal{E} \sin (\omega t+\phi), \pm \Delta_{\mathrm{SO}}\right)
$$

are the Rabi vectors, and $\boldsymbol{\sigma}=\left(\sigma_{x}, \sigma_{y}, \sigma_{z}\right)$ is the Pauli vector operator that acts on the chiral degrees of freedom. $\omega, \phi$, and $\mathcal{E}$ are the angular frequency, phase, and amplitude of the oscillating electric field, respectively, and $\pm \Delta_{\mathrm{SO}}$ are the zero-field energy splittings between the chiral states, when the spin is in state $\left| \pm \frac{1}{2}\right\rangle$.

The chirality dynamics conditioned on the spin state is described by

$$
\frac{d}{d t} \mathbf{s}_{ \pm}(t)=\boldsymbol{\Omega}_{ \pm}(t) \times \mathbf{s}_{ \pm}(t)
$$




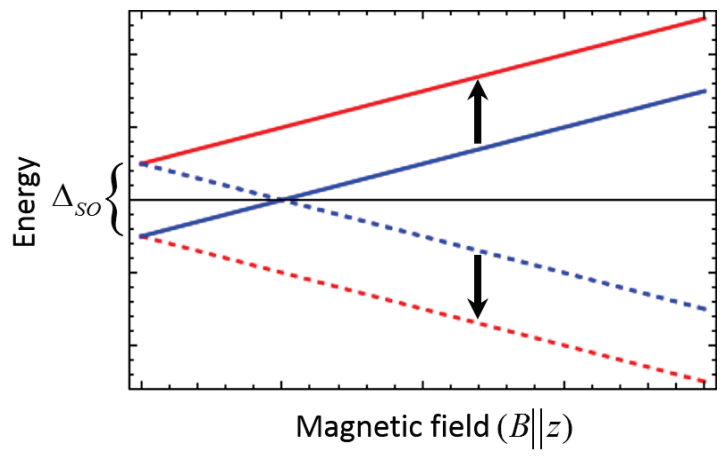

FIG. 2. (Color online) Schematic diagram of electric-fieldinduced transitions between states of opposite chirality in the ground-state manifold of a triangular MM, with the zero-field splitting $\Delta_{\text {SO }}$ due to the Dzyaloshinsky-Moriya interaction. Red solid (dashed) lines represent the states with $\chi=+1$ and $S_{z}=+\frac{1}{2}\left(-\frac{1}{2}\right)$, and blue solid (dashed) lines represent the states with $\chi=-1$ and $S_{z}=+\frac{1}{2}\left(-\frac{1}{2}\right)$.

where $\mathbf{s}_{ \pm}(t)$ are the instantaneous Bloch vectors parametrizing the conditional chiral density operators

$$
\rho_{ \pm}(t)=\frac{1}{2}\left[I+\mathbf{s}_{ \pm}(t) \cdot \boldsymbol{\sigma}\right] \otimes\left| \pm \frac{1}{2}\right\rangle\left\langle \pm \frac{1}{2}\right|,
$$

given by solving the equations of motion

$$
i \hbar \frac{d}{d t} \rho_{ \pm}(t)=\left[H_{\mathrm{eff}}(t), \rho_{ \pm}(t)\right]
$$

In the rotating frame with angular frequency $\omega$ around the $z$-axis, Eq. (10) is equivalent to

$$
\frac{d}{d t} \mathbf{s}_{ \pm}^{\prime}(t)=\mathbf{\Omega}_{ \pm}^{\prime} \times \mathbf{s}_{ \pm}^{\prime}(t)
$$

with the time-independent Rabi vectors

$$
\hbar \boldsymbol{\Omega}_{ \pm}^{\prime}=\left(p \mathcal{E} \cos \phi, p \mathcal{E} \sin \phi, \pm \Delta_{\mathrm{SO}}-\hbar \omega\right) .
$$

If the Bloch vector $\mathbf{s}_{+}^{\prime}\left(\mathbf{s}_{-}^{\prime}\right)$ is initially aligned with $\boldsymbol{\Omega}_{+}^{\prime}$ $\left(\boldsymbol{\Omega}_{-}^{\prime}\right)$, it remains aligned with $\boldsymbol{\Omega}_{+}^{\prime}\left(\boldsymbol{\Omega}_{-}^{\prime}\right)$ under an adiabatic variation of the electric field parameters $\mathcal{E}$ and $\phi$. Therefore, since we can realize different vectors $\boldsymbol{\Omega}_{+}^{\prime}\left(\boldsymbol{\Omega}_{-}^{\prime}\right)$ by controlling the electric field parameters, we can adiabatically move the Bloch vector $\mathbf{s}_{+}^{\prime}\left(\mathbf{s}_{-}^{\prime}\right)$ into different positions on the Bloch sphere.

\section{GEOMETRIC PHASE SHIFT}

In the absence of an electric field, the energy eigenstates of the Hamiltonian in Eq. (8) are the basis states given in Eq. (3). Hence, the Bloch vector $\mathbf{s}_{ \pm}^{\prime}$ corresponding to each energy eigenstate is either parallel or antiparallel to $\boldsymbol{\Omega}_{ \pm}^{\prime}$. This implies that by varying the effective Hamiltonian in Eq. (8) adiabatically by slowly changing the parameters of the electric field, we can let each energy eigenstate evolve in a cyclic fashion. Figure 3 depicts the cyclic evolution $\mathcal{C}$ accomplished first by slowly increasing

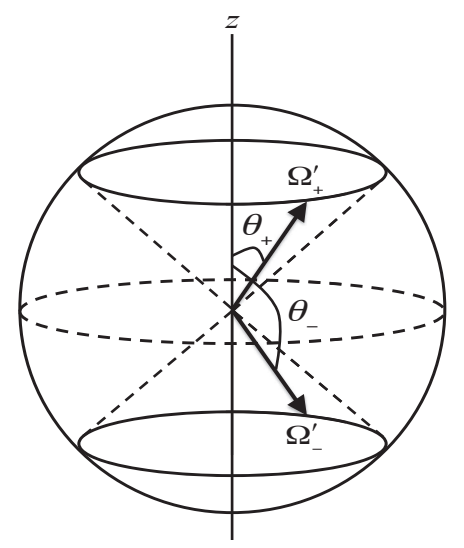

FIG. 3. Schematic picture of the paths in parameter space corresponding to cyclic evolutions of conditional chiral states in the adiabatic limit. Depending on whether the initial chiral Bloch vector $\mathbf{s}_{ \pm}^{\prime}$ is parallel or anti-parallel to the initial Rabi vector $\boldsymbol{\Omega}_{ \pm}^{\prime}$, the Bloch vector remains parallel or anti-parallel, respectively, to the Rabi vector along the adiabatic evolution. Thus, the polar angle $\tilde{\theta}_{ \pm}$between the Bloch vector $\mathbf{s}_{ \pm}^{\prime}$ and the $z$-axis throughout the precession would be $\theta_{ \pm}$or $\pi-\theta_{ \pm}$ depending on parallel condition between the initial Bloch and Rabi vectors.

the field amplitude from zero to $\mathcal{E}$, then precessing the field around the $z$-axis by slowly varying the phase $\phi$, and finally switching the field off by slowly decreasing its amplitude to zero.

In such an adiabatic evolution, each energy eigenstate accumulates a Berry phase, being proportional to the solid angle subtended by its corresponding path on the Bloch sphere representing the chirality state space $\operatorname{Span}\{| \pm 1\rangle\}$. The associated geometric phases can be calculated by specifying the polar angle $\tilde{\theta}_{ \pm}$between the Bloch vector $\mathbf{s}_{ \pm}^{\prime}$ and the $z$-axis throughout the precession. In the adiabatic regime, this angle is given by the angle between the Rabi vector $\boldsymbol{\Omega}_{ \pm}^{\prime}$ and the $z$-axis, i.e.,

$$
\cos \theta_{ \pm}=\frac{ \pm \Delta_{\mathrm{SO}}-\hbar \omega}{\sqrt{\left( \pm \Delta_{\mathrm{SO}}-\hbar \omega\right)^{2}+(p \mathcal{E})^{2}}}
$$

Note that, depending on whether the initial Bloch vector is parallel or anti-parallel to the initial Rabi vector, the polar angle $\tilde{\theta}_{ \pm}$is $\theta_{ \pm}$or $\pi-\theta_{ \pm}$, respectively (see Fig. 3). Along this evolution, the instantaneous energy eigenstates can be parametrized as

$$
\begin{aligned}
\left|\Psi_{ \pm}(\zeta, \xi)\right\rangle= & {\left[\cos (\zeta / 2)|+1\rangle+e^{i \xi} \sin (\zeta / 2)|-1\rangle\right] } \\
\otimes & \left. \pm \frac{1}{2}\right\rangle
\end{aligned}
$$

where $\zeta$ varies smoothly between zero and the polar angle $\tilde{\theta}_{ \pm}$, and $\xi$ changes slowly between zero and $2 \pi$. Using this 
parametrization, one can calculate the Berry phases as

$$
\begin{aligned}
\gamma_{ \pm} & =i \oint_{\mathcal{C}}\left\langle\Psi_{ \pm}(\zeta, \xi)|d| \Psi_{ \pm}(\zeta, \xi)\right\rangle \\
& =i \int_{0}^{2 \pi}\left\langle\Psi_{ \pm}\left(\tilde{\theta}_{ \pm}, \xi\right)\left|\frac{d}{d \xi}\right| \Psi_{ \pm}\left(\tilde{\theta}_{ \pm}, \xi\right)\right\rangle d \xi \\
& =-\pi\left(1-\cos \tilde{\theta}_{ \pm}\right) .
\end{aligned}
$$

Considering the fact that the polar angles $\tilde{\theta}_{ \pm}$depend on the orientation of initial Bloch vectors we obtain the following Berry phases

$$
\begin{aligned}
& \gamma_{+1,+\frac{1}{2}}=-\gamma_{-1,+\frac{1}{2}}=\gamma_{+}=-\pi\left(1-\cos \theta_{+}\right), \\
& \gamma_{-1,-\frac{1}{2}}=-\gamma_{+1,-\frac{1}{2}}=\gamma_{-}=-\pi\left(1-\cos \theta_{-}\right)
\end{aligned}
$$

with corresponding dynamical phases

$$
\begin{aligned}
& \delta_{ \pm 1,+\frac{1}{2}}=\frac{-1}{2 \hbar} \int_{0}^{T}\left[g_{\perp} B_{z} \pm \sqrt{\left(\Delta_{\mathrm{SO}}-\hbar \omega\right)^{2}+(2 p \mathcal{E})^{2}}\right] d t \\
& \delta_{ \pm 1,-\frac{1}{2}}=\frac{1}{2 \hbar} \int_{0}^{T}\left[g_{\perp} B_{z} \pm \sqrt{\left(\Delta_{\mathrm{SO}}+\hbar \omega\right)^{2}+(2 p \mathcal{E})^{2}}\right] d t .
\end{aligned}
$$

Clearly, the cyclic evolution $\mathcal{C}$ yields the unitary phase transformation

$$
|x, y\rangle \longrightarrow e^{i\left(\gamma_{x, y}+\delta_{x, y}\right)}|x, y\rangle, \quad x, 2 y= \pm 1,
$$

of the spin-chirality basis vectors.

In order to realize purely geometric phase shifts, it is necessary to eliminate the dynamical phases $\delta_{x, y}$. This can be achieved by using a technique known as spinecho ${ }^{34.35}$. In this procedure, we apply the cyclic evolution $\mathcal{C}$ combined with fast $\pi$ transformations, which simply flip the spin or chiral basis states by applying in the molecular plane a pulsed magnetic or electric field, respectively. Explicitly, we let the system evolve through the following compound evolution

$$
C_{\text {net }}: \quad \mathcal{C} \rightarrow \pi_{2} \rightarrow \mathcal{C} \rightarrow \pi_{1} \rightarrow \mathcal{C}^{-1} \rightarrow \pi_{2} \rightarrow \mathcal{C}^{-1} \rightarrow \pi_{1}
$$

where $\pi_{2}\left(\pi_{1}\right)$ is a fast spin (chiral) flip transformation, and the path $\mathcal{C}^{-1}$ in parameter space is the same as $\mathcal{C}$ described above, but in the reverse direction. The net effect of this compound transformation would be that the dynamical phases are all canceled out and we are only left with geometric phase factors. Thus, the net unitary transformation is given by

$$
\begin{aligned}
U\left(C_{\text {net }}\right)= & \left(e^{i 2 \Delta \gamma}|+1\rangle\langle+1|\right. \\
& \left.+e^{-i 2 \Delta \gamma}|-1\rangle\langle-1|\right) \otimes \hat{1}_{\text {spin }},
\end{aligned}
$$

purely dependent on the Berry phase shift

$$
\begin{aligned}
\Delta \gamma= & \gamma_{+}-\gamma_{-}=\pi\left(\cos \theta_{+}-\cos \theta_{-}\right) \\
= & \pi\left(\frac{\Delta_{\mathrm{SO}}-\hbar \omega}{\sqrt{\left(\Delta_{\mathrm{SO}}-\hbar \omega\right)^{2}+(p \mathcal{E})^{2}}}\right. \\
& \left.+\frac{\Delta_{\mathrm{SO}}+\hbar \omega}{\sqrt{\left(\Delta_{\mathrm{SO}}+\hbar \omega\right)^{2}+(p \mathcal{E})^{2}}}\right),
\end{aligned}
$$

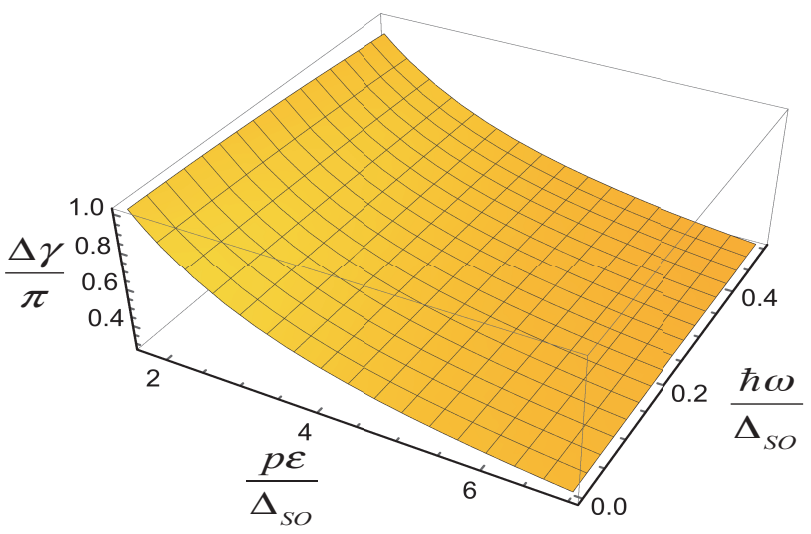

FIG. 4. Geometric phase shift $\Delta \gamma$ as a function of $\frac{\hbar \omega}{\Delta_{\mathrm{SO}}}$ and $\frac{p \mathcal{E}}{\Delta_{\mathrm{SO}}}$, where $\omega$ is the angular frequency of the applied electric field, $p$ is the strength of the spin-electric coupling, $\mathcal{E}$ is the applied field amplitude, and $\Delta_{\mathrm{SO}}$ is the zero-field energy splitting of the chiral states.

and acting nontrivially only on the chiral degree of freedom. This follows from the fact that the cyclic adiabatic evolution in opposite directions induce the same dynamical phases but Berry phases with opposite signs. The unitary operator $U\left(C_{\text {net }}\right)$ can be viewed as a geometric phase-shift gate ${ }^{34}| \pm 1\rangle \mapsto e^{ \pm i 2 \Delta \gamma}| \pm 1\rangle$ acting on a chiral qubit encoded in the ground state manifold of the MM.

Figure 4 shows that by careful control of the electric field amplitude any Berry phase shift associated with the chiral qubit can be realized. It is worth noticing that $\Delta \gamma$ is a Lorentzian shaped phase shift with no local extrema and is independent of the applied external magnetic field. In the adiabatic limit $\omega \rightarrow 0$, we obtain

$$
\lim _{\omega \rightarrow 0} \Delta \gamma=2 \pi \frac{\Delta_{\mathrm{SO}}}{\sqrt{\left(\Delta_{\mathrm{SO}}\right)^{2}+(p \mathcal{E})^{2}}}
$$

which establishes a fundamental relation between three quantities: Berry phase $\Delta \gamma$, the strength of the spinelectric coupling $p$, and the zero-field energy splitting $\Delta_{\mathrm{SO}}$.

The relation in Eq. (24) provides in principle a method for obtaining an estimate of the ratio $\Delta_{\mathrm{SO}} / p$ of the two primary intrinsic quantities regulating the spin-electric coupling mechanism in these MMs. By measuring the geometric Berry phase $\Delta \gamma$ as a function of the external electric field amplitude $\mathcal{E}$, the ratio can be evaluated.

The geometric phase can be measured interferometrically, by canceling the dynamical phases picked up along different interference pathways. In the MM case, one may measure the Berry phase $\Delta \gamma$ by using a spin-echo interference setting, where the spin degree of freedom plays the role of the interferometer arms. One way to realize this would be to initialize the interferometer in an eigenstate of $\sigma_{x} \otimes \hat{1}_{\text {spin }}$ with, e.g., a short $\frac{\pi}{2}$ electric field pulse applied in the plane of the molecule. The system subsequently evolves through the spin echo compound evolution $C_{\text {net }}$ described above, by carefully controlling 
the external fields. Right after completing the spin echo sequence, another $\frac{\pi}{2}$ electric field pulse is applied followed by measuring the chirality of the output. One finds the probabilities

$$
\begin{aligned}
& P(\text { chirality }=+1)=\cos ^{2}(2 \Delta \gamma), \\
& P(\text { chirality }=-1)=\sin ^{2}(2 \Delta \gamma)
\end{aligned}
$$

from which the Berry phase shift can be extracted. A direct measurement of the chirality in these MMs is a non-trivial task. However, as it was pointed by Khomskii et al. ${ }^{15,36}$, the chiral GS states are characterized by the presence of spontaneous orbital currents, giving rise to magnetic orbital moments proportional to the chirality. Therefore it is in principle possible to determine the state chirality by carrying out a measure of the orbital moment via, e.g., Stern-Gerlach-type experiments.

We conclude this section with two remarks on the effect of the external magnetic field. First, we note that in the conditional chiral spin dynamics described above, a static magnetic field perpendicular to the plane of the MM plays only the passive role of splitting the two chiral components with opposite spin quantum numbers. Importantly, in creating ensembles of solid-state molecular spin qubits this function of the magnetic field is one possible solution to the unwanted long-range magnetic dipolar interaction, which is one of the strongest source of decoherence in crystals of quantum molecular magnets ${ }^{37}$. However, a large magnetic field also renders coherent manipulations of spin qubits impractical. An alternative way of controlling spin decoherence of a spin qubits in a solid-state environment involves diluting the concentration of spin, but this has the drawback of weakening the nearest-neighbor spin interaction, needed for qubit entanglement. Quite recently a novel and more ingenious technique based on "atomic clock transitions" has been demonstrated $^{38}$. In our case this is not an issue, since the chiral qubit of a triangular MM is entirely manipulated by the electric field, while a constant magnetic field is still used to freeze out one of the two spin components.

The second remark concerns the effect of a component of a constant magnetic field in the plane of the molecule. When this is present, spin-up and spin-down states are coupled by the transverse field $B_{x y}$. Nevertheless, if $B_{x y} \ll B_{z}$, then the eigenstates of the effective Hamiltonian are still of predominant spin character, with a small admixture of the opposite spin contribution. When a time-dependent field is applied, we can imagine repeating the same analysis of the chiral state dynamics induced by the spin-electric coupling. Now, however, since the states are no longer pure spin state, the electric field couples chiral states of opposite chirality and predominately opposite spin. Using two states of opposite chirality and opposite spin to encode a qubit has the advantage that transitions between them can be more easily read out via a detection of the spin flip 39 .

\section{SUMMARY}

In summary, we have investigated the Berry phase effect in the system of triangular antiferromagnetic molecular magnets (MMs). We have demonstrated the existence of a Berry phase associated with the chiral degree of freedom (chiral qubit) of the system that can be measured by using spin-echo techniques. We show that a nontrivial Berry phase shift can be realized even with an in-plane external field, due to the presence of a spin-orbit term that couples the chiral and spin degrees of freedom. We have derived a unifying relation between the Berry phase, as a function of electric field and the two primary intrinsic quantities in the MM being the spin-orbit coupling strength and the electric dipole moment. In this way, the ratio between these quantities can be estimated by measuring the Berry phase shift on the chiral degree of freedom. Furthermore, by considering the two chiral states as defining a qubit embedded in the ground state manifold of the triangular MM, the Berry phase effect can be interpreted as a single-qubit geometric phase shift gate. The research of this paper provides an experimental testbed for exploring the physical nature of the Berry's phase effect in solid state systems.

We have confined ourselves to the study of the chiral dynamics of isolated triangular MMs. As we mentioned in the Introduction, these systems can be possibly addressed in molecular beam experiments, similar to the ones realized in Ref. 22. An alternative experimental realization of the effect studied in this paper could possibly involve the functionalization of the MMs onto an appropriate surface/substrate, which are then electrically addressed by a nearby STM tip and electric gates. The choice of the surface is crucial. First of all the $D_{3 h}$ symmetry of the molecule has to be preserved in such a way that the simple theoretical model discussed above is applicable. Graphene and boron nitride are both substrates that display the correct crystal symmetry. Secondly, unwanted charge-transfer effects between the molecule and substrate that would mask and complicate the realization of the spin-electric coupling must be avoided or controlled. This is in fact a challenging task. Ongoing firstprinciples calculations ${ }^{32}$ of $\mathrm{Cu}_{3}$ and $\mathrm{V}_{3}$ MMs on graphene and boron nitride substrates can provide useful hints on the effect of the environment on the chiral GS manifold and the spin-electric coupling in these antiferromagnetic triangular molecular magnets. Another interesting possibility to realize experimentally the effects proposed in this paper consists in utilizing self-regulated atom trapping in open nanocorrals to built triangular clusters on surfaces with atomic-level precision and without the need for ligands 40 .

\section{ACKNOWLEDGMENTS}

One of us (C.M.C.) would like to thank Md F. Islam and M.R. Pederson for interesting discussions on 
the first-principles studies of triangular antiferromagnetic molecules. This work was supported by Department of Mathematics at University of Isfahan (Iran), and Department of Physics and Electrical Engineering at Linnaeus University (Sweden). C.M.C. acknowledges finan- cial support from the Swedish Research Council (VR) through Grant No. 621-2014-4785. E.S. acknowledges financial support from the Swedish Research Council (VR) through Grant No. D0413201.
1 M. V. Berry, Proc. Roy. Soc. London, Ser. A 392, 45 (1984).

2 Y. Aharonov and J. Anandan, Phys. Rev. Lett. 58, 1593 (1987).

3 J. Anandan, Phys. Lett. A 133, 171 (1988).

${ }^{4}$ F. Wilczek and A. Zee, Phys. Rev. Lett. 52, 2111 (1984).

5 A. Uhlmann, Rep. Math. Phys. 24, 229 (1986).

${ }^{6}$ E. Sjöqvist, A. K. Pati, A. Ekert, J. S. Anandan, M. Ericsson, D. K. L. Oi, and V. Vedral, Phys. Rev. Lett. 85, 2845 (2000).

7 A. Tomita and R. Y. Chiao, Phys. Rev. Lett. 57, 937 (1986).

8 H. von Busch, V. Dev, H.-A. Eckel, S. Kasahara, J. Wang, W. Demtröder, P. Sebald, and W. Meyer, Phys. Rev. Lett. 81, 4584 (1998).

9 Y. Zhang, Y.-W. Tan, H. L. Stormer, and P. Kim, Nature (London) 438, 201 (2005).

10 D. Xiao, M. C. Chang, and Q. Niu, Rev. Mod. Phys. 82, 1959 (2010).

${ }^{11}$ L. Bogani and W. Werndorfer, Nat. Mat. 7, 179 (2008).

12 A. V. Chumak, V. I. Vasyuchka, A. A. Serga and B. Hillebrands, Nat. Phys. 11, 453 (2015).

13 D. Gatteschi, R. Sessoli, and J. Villain, Molecular Nanomagnets, Oxford University Press, Oxford, U.K., 2006.

14 K.-Y. Choi, Y. H. Matsuda, H. Nojiri, U. Kortz, F. Hussain, A. C. Stowe, C. Ramsey, and N. S. Dalal, Phys. Rev. Lett. 96, 107202 (2006).

15 L. N. Bulaevskii, C. D. Batista, M. V. Mostovoy, and D. I. Khomskii, Phys. Rev. B 78, 024402 (2008).

16 M. Trif, F. Troiani, D. Stepanenko, and D. Loss, Phys. Rev. Lett. 101, 217201 (2008).

17 M. Trif, F. Troiani, D. Stepanenko, and D. Loss, Phys. Rev. B 82, 045429 (2010).

18 S. Thiele, F. Balestro, R. Ballou, S. Klyatskaya, M. Ruben, and W. Wernsdorfer, Science 344, 1135 (2014).

19 E. Kawakami1, P. Scarlino1, D. R. Ward, F. R. Braakman1, D. E. Savage, M. G. Lagally, Mark Friesen, S. N. Coppersmith, M. A. Eriksson, and L. M. K. Vandersypen, Nat. Nanotechnol. 9, 666 (2014).

20 A. Laucht, J. T. Muhonen, F. A. Mohiyaddin, R. Kalra, J. P. Dehollain, S. Freer, F. E. Hudson, M. Veldhorst, R. Rahman, G. Klimeck, K. M. Itoh, D. N. Jamieson, J. C. McCallum, A. S. Dzurak, and A. Morello, Sci. Adv. 1, e1500022 (2015).

21 B. E. Kane, Nature (London) 393, 133 (1998).

${ }^{22}$ W. Jin, M. Becherer, D. Bellaire, G. Lefkidis, M. Gerhards, and W. Hübner, Phys. Rev. B 89, 144409 (2014).
23 D. Chaudhuri, W. Jin, G. Lefkidis, and W. Hübner, J. Chem. Phys. 143, 174303 (2015).

24 SMMs are an important class of MMs characterized by a combination of a large ground-state spin and a large magnetic anisotropy of the Ising (easy-axis) type 13 . Famous examples are $\mathrm{Mn}_{12}$-acetate and $\mathrm{Fe}_{4}$ complexes ${ }^{13}$. An interesting example of a triangular SMM based on lanthanide atoms, e.g., Dysprosium, instead of the more common transition metal compounds was analyzed in Ref. 25 .

25 J. Tang et al., Angew. Chem. Int. Ed. , 45, 1729 (2006).

26 I. Dzyaloshinsky, J. Phys. Chem. Solids 4, 241 (1958).

27 T. Moriya, Phys. Rev. 120, 91 (1960).

28 Both available experimental studies on triangular molecules, e.g. $\mathrm{Cu}_{3}{ }^{14}$, and considerable computational work done within density functional theory (DFT) ${ }^{29,30}$ seem to indicate that the GS is indeed a doubly degenerate $S=\frac{1}{2}$ doublet, with degeneracy lifted by a spin-orbit interaction and possibly by the Jahn-Teller effect, as detailed above. However, the nature of the (super) exchange coupling in these molecular systems is complex, since the effective interaction between magnetic ions is mediated via long bridges of non-magnetic atoms. We cannot exclude that in some cases, possibly for systems with strong correlations, effective exchange constants with ferromagnetic (FM) coupling might become dominant, leading to the highest multiplicity state being the GS.

29 J. F. Nossa, M. F. Islam, C. M. Canali, and M. R. Pederson, Phys. Rev. B 85, 085427 (2012).

30 M. F. Islam, J. F. Nossa, C. M. Canali, and M. R. Pederson, Phys. Rev. B 82, 155446 (2010).

31 T. Murao, Phys. Lett. 49 A, 33 (1974).

32 M. F. Islam and C. M. Canali, unpublished (2016).

33 F. S. Ham, Phys. Rev. Lett. 58, 725 (1987).

34 A. Ekert, M. Ericsson, P. Hayden, H. Inamori, J. A. Jones, D. K. L. Oi, and V. Vedral, J. Mod. Opt. 47, 2501 (2000).

35 J. A. Jones, V. Vedral, A. Ekert, and G. Castagnoli, Nature (London) 403, 869 (2000).

36 D. I. Khomskii, J. Phys. Condens. Matter 22164209 (2010).

37 S. Takahashi, I. S. Tupitsyn, J. van Tol, C. C. Beedle, D. N. Hendrickson, and P. C. E. Stamp, Nature (London) 476, 76 (2011).

38 M. Shiddiq, D. Komijani, Y. Duan, A. Gaita-Arino, E. Coronado, and S. Hill, Nature (London) 531, 348 (2016).

39 F. Meier, J. Levy, and D. Loss, Phys. Rev. B 68, 134417 (2003).

40 R. X. Cao, Z. Liu, B. F. Miao, L. Sun, D. Wu, B. You, S. C. Li, W. Zhang, A. Hu, S. D. Bader, and H. F. Ding, Phys. Rev. B 90, 045433 (2014). 